\title{
Testing Secluded Dark Matter Models with Neutrino Telescopes
}

\author{
A. Herrero \\ Institut de Matmàtica Multidisciplinar, Universitat Politècnica de València, Spain
}

\begin{abstract}
The existence of Dark Matter (DM) in the Universe is supported by cosmological and astrophysical evidences. Observations indicate that DM is non-baryonic, non-relativistic and not subject to electromagnetic interactions, but its nature is still unknown. A hypothesis can be based on the idea that DM is "secluded" from Standard Matter particles and that the annihilation is only possible through a metastable mediator. Neutrino telescopes may be efficient tools to test Secluded Dark Matter (SDM) models, such as dark photon models, by means of different searches in the Sun, Earth and Galactic Center. In this work, searches for SDM in neutrino telescopes are discussed, especially the analysis done of the 79-string IceCube public data to test SDM models. In this analysis no significant excess over background is observed and constraints on the parameters of the models are derived. The analysis is also used to constrain the dark photon model.
\end{abstract}

Keywords: Neutrino Telescopes, Secluded dark matter, Dark photon model

DOI: 10.31526/ACP.NDM-2020.25

\section{INTRODUCTION}

There is strong cosmological and astrophysical evidence for the existence of Dark Matter in the Universe. Observations indicate that DM is about a quarter of the total mass-energy of the Universe, and it is non-baryonic, non-relativistic and not subject to electromagnetic interactions, but its nature is still unknown [1]. The Weakly Interacting Massive Particle (WIMP) model has been the most popular explanation for it and a big effort has been done in the last decades to test it, resulting in no detection and severe constraints. An alternative hypothesis is based on the idea that DM may live in a dark sector with its own forces, so it is "secluded" from Standard Matter (SM) particles and that the annihilation is only possible through a metastable mediator, which subsequently decays into SM states [2]. This kind of models can retain the thermal relic WIMP DM scenario while at the same time explain other 'anomalous' observations, such as the positron-electron ratio observed by AMS-II [3].

Neutrino telescopes may be efficient tools to test Secluded Dark Matter models, such as dark photon model, by means of different searches in the Sun [4, 5], Earth [6] and Galactic Center [7], which could complement constraints from direct detection, accelerators, astrophysics and indirect detection with other messengers, such as gamma rays [8] or antiparticles [9].

In this paper the searches for SDM in neutrino telescopes will be discussed, especially the searches using the Sun as source. First, we review the search done by ANTARES Collaboration [4]. Afterwards, the analysis done of the 79-string IceCube public data to test SDM models [5] is discussed. In this analysis no significant excess over background was observed and constraints on the parameters of the models were derived. The analysis is also used to constrain the dark photon model in the region of the parameter space with dark photon masses between 0.22 and $\sim 1 \mathrm{GeV}$ and a kinetic mixing parameter of about $10^{-9}$, which is a difficult region to explore with other methods. Some prospects for future searches in neutrino detectors being built are also addressed.

\section{ANTARES STUDY}

Secluded dark matter model is proposed as a modification of the dark matter model in which the dark matter and normal matter are secluded interacting only through a mediator. This mediator could be some new gauge boson from the dark sector or any other candidate. The simplest situation is to consider dark matter that annihilates into mediators, which can decay for example into two muons. Note that if the mass of the dark matter is greater than the mediator mass then the dark matter would be leptophilic. Considering this simplest picture, celestial bodies, such as the Sun, that capture dark matter, are sources of mediators. So, looking at these bodies, if the lifetime of the mediator is long enough it could decay out of the Sun or even near the Earth, producing two collinear muons, pions or even neutrinos directly, see Figure 1.

Under all these considerations, ANTARES looked for SDM in the direction of the Sun taking into account three situations: 1) the mediator decays into two parallel muons near the Earth and these muons are detected by the telescope; 2 ) the mediator decays into muons in its path to the Earth, which also decay and produce neutrinos; and 3) the case in which the mediator directly decays into neutrino.

The ANTARES study was the first conducted by a neutrino telescope Collaboration that leads to important constraints in the SDM models [4]. The no detection of dimuons and the no observation of an excess of neutrinos resulted in exclusion regions in annihilation of Dark Matter in the Sun versus the length of the mediator decay, for both possibilities studied: mediator decaying into dimuons and mediator decaying directly into neutrino. 


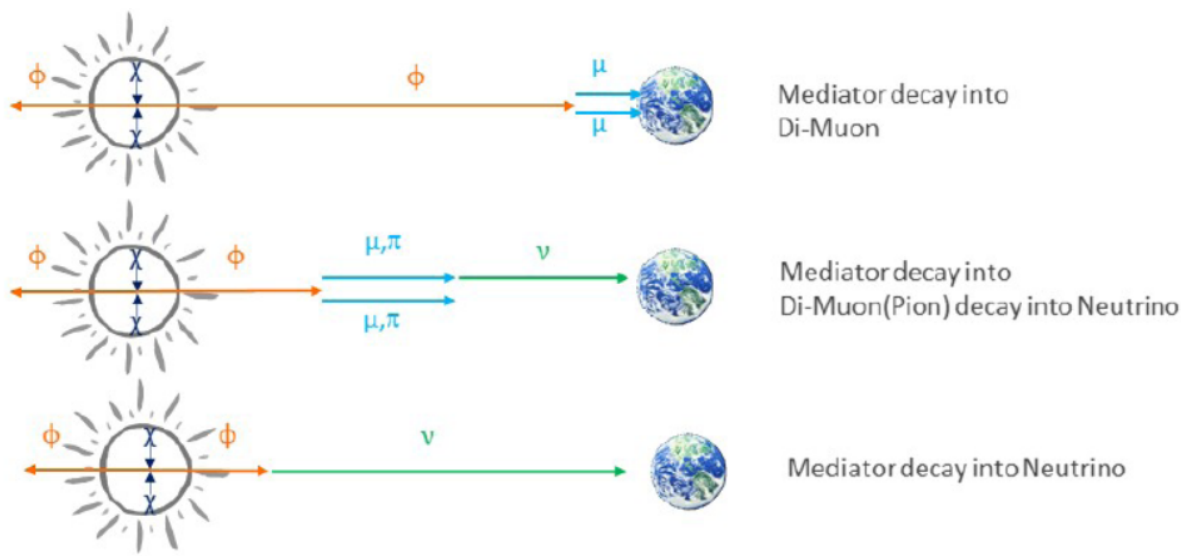

FIGURE 1: Schematic view of the SDM searches towards the Sun in neutrino telescopes. Adapted from [10].

ANTARES results were also interpreted as limits in the nucleon-WIMP cross section interaction for both cases spin dependent and spin independent. Assuming mediator decay lengths larger than the radius of the Sun and smaller than the Sun-Earth distance, the resulting limits were much more stringent for large dark matter masses, $\gtrsim 100 \mathrm{GeV}$ for spin dependent and $\gtrsim 5 \mathrm{TeV}$ for spin independent interaction.

\section{ANALYSIS WITH THE 79-STRING ICECUBE PUBLIC DATA}

In this section, the analysis done for SDM search using the public data of the 79 strings of IceCube Dark Matter search in the Sun is reviewed [5,11]. IceCube is a large high-energy neutrino observatory located in the ice of Antarctica [12].

In the analysis, the Winter High-Energy set of 79-string IceCube public data with 136 days is used. It contains the information of the reconstructed events: direction, energy parameters and so on; the background probability density distribution functions in 1 degree bins with respect to the direction of the Sun; and the efficiency of the detector in terms of the effective areas and effective volume, as well as the energy response. First, a sensitivity study was made by selecting the best cuts in a blind way taking into account the response of the detector and the background. The energy information was not taken into consideration since its relevance was smaller than the other parameters, and thus, was not considered for the cuts. The final angle cut was selected to have the best sensitivity using Feldmann-Cousins statistics. It was obtained that the final flux sensitivity for mediators decaying into muons, pions and neutrinos was much better than the obtained in the previous ANTARES results. The number of observed events were compatible with the expected background and upper limits were derived, setting constraints to the SDM models, similarly to the ANTARES study, but with a clear improvement in the exclusion regions and in the WIMP-proton cross section for spin dependent and spin independent interaction. For large DM mass and sufficiently long lifetime of the mediator, the limits for the spin-dependent case are much better than those derived by direct detection experiments [13, 14].

Recently, a similar study using also the 79-string IceCube public data has been done by Niblaeus el al. [15] obtaining similar exclusion regions, but slightly less constraining regions. The difference between both studies is not completely clear, it may be due to a more detailed treatment in the propagation of particles in the last study, or in differences in the statistics treatment.

\section{CONSTRAINTS TO DARK PHOTON MODEL}

In this section we review the work [5] interpreted in terms of the dark photon model. In this model there is a dark photon that kinetically mixes with the standard model photon. Feng et al. [6] proposed to use neutrino telescope to test the dark photon model by detecting dimuons or showers from dark matter captured in the Earth. For testing the model they also proposed to use AMS to detect positrons from dark photons coming from the Sun [9]. The same idea but using the resulting neutrinos coming from the Sun is used in [5]. There are four parameters in the model: the dark photon mass $m_{A^{\prime}}$, the dark matter mass, $m_{\chi}$, the kinetic mixing parameter, $\varepsilon$, that control the interaction with ordinary matter and the dark fine structure constant $\alpha_{X}$, that control the interaction with dark matter. Typically, the $\alpha_{X}$ is also fixed by requiring the dark matter particle to saturate the observed dark matter density through thermal freeze out, so $\alpha_{X}=\alpha_{X}^{\text {th }} \simeq 0.035\left(m_{\chi} / \mathrm{TeV}\right)$. Alternatively, the maximum allowed coupling is set by bounds on distortions to the cosmic microwave background, and then $\alpha_{X}^{\max } \simeq 0.17\left(m_{\chi} / \mathrm{TeV}\right)^{1.61}$ for the mass range considered [6]. In this case, the decay length of the mediator is not free but dependent on the other parameters. The Branching ratio into SM states is also given by theory. By considering the decay length and the Branching ratios given by the model, it is quite straightforward to transform the previous constraints into bounds to the dark photon model and its parameter space region. It is also possible to interpret the no detection in terms of constraints to the spin independent WIMP-nucleon cross section interaction, as shown in Figure 2. As it can be observed from the figure, there are some unconstrained regions that can be constrained using existing 
and, especially, future neutrino telescopes. The arrows indicate regions that could be explored using future detectors, such as KM3NeT/ORCA and PINGU for intermediate DM mass, and KM3NeT/ARCA and IceCube-Gen2 for large DM mass [16, 17].

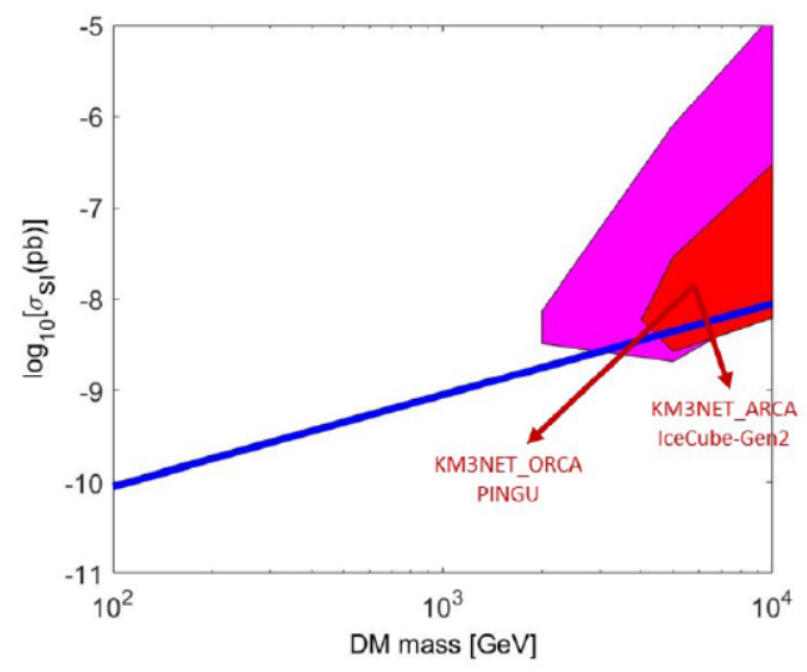

FIGURE 2: Constraints in the $\left(m_{X}, \sigma\right)$ plane from [5] for a dark photon mass of $250 \mathrm{MeV}$ and assuming $\alpha_{X}=\alpha_{X}^{\text {th }}\left(\right.$ red) or $\alpha_{X}^{\text {max }}$ (magenta). The bounds from Xenon 1ton [18] are also shown (blue line).

\section{CONCLUSIONS}

Different secluded dark matter models have been proposed to explain dark matter and other experimental anomalies observed, being the dark photon model one of the most promising. As shown, it is possible to test some of the parameters of these models with searches in neutrino telescopes. For instance, the search for SDM in the Sun using the 79-string IceCube public data has set relevant constraints both for generic models and for the particular Dark Photon model, starting to explore regions in a complementary way to other techniques. This will also be the case in the near future with the construction of new detectors KM3Net-ARCA/ORCA and IceCube-Gen2/PINGU, where the combination of different kind of detectors and different sources (Earth, Sun and Galactic Center) opens up different windows of possibilities in this topic.

\section{ACKNOWLEDGEMENTS}

We acknowledge the financial support of Plan Estatal de Investigación, ref. FPA2015-65150-C3-2-P (MINECO/FEDER).

\section{References}

[1] G. Bertone, D. Hooper and J. Silk, Phys. Rep. 405, 279 (2005).

[2] M. Pospelov, A. Ritz and M. B. Voloshin, Phys. Lett. B 662, 53 (2008).

[3] L. Accardo, et al., Phys. Rev. Lett. 113, 121101 (2014).

[4] S. Adrián-Martínez et al.,JCAP 05, 016 (2016).

[5] M. Ardid et al., JCAP 04, 010 (2017).

[6] J.L. Feng, J. Smolinsky and P. Tanedo, Phys. Rev. D 93, 015014 (2016).

[7] ANTARES Coll. and F. Sala, PoS(ICRC2019) 519.

[8] A. Albert et al. (HAWC Collaboration), Phys. Rev. D 98, 123012.

[9] J.L. Feng, J. Smolinsky and P. Tanedo, Phys. Rev. D 93, 115036 (2016).

[10] S. Adrián-Martínez, Ph. D. Thesis, Universitat Politècnica de València (2015).

[11] M. G. Aartsen et al., JCAP 04, 022 (2016).

[12] F. Halzen and S.R. Klein, Rev. Sci. Instrum. 81, 081101 (2010).

[13] C. Amole et al., Phys. Rev. Lett. 118, 251301 (2017).

[14] C. Amole et al., Phys. Rev. D 100, 022001 (2019).

[15] Carl Niblaeus et al, JCAP 11, 011 (2019).

[16] S. Adrián-Martínez et al., J. Phys. G 43 43, 084001 (2016).

[17] M. G. Aartsen et al., Physical Review D 101, 032006 (2020).

[18] E. Aprile et al., Phys. Rev. Lett. 121, 111302 (2018). 